\title{
Functional inequalities for Gaussian convolutions of compactly supported measures: Explicit bounds and dimension dependence
}

\author{
JEAN-BAPTISTE BARDET ${ }^{1}$, NATHAËL GOZLAN ${ }^{2}$, \\ FLORENT MALRIEU ${ }^{3}$ and PIERRE-ANDRÉ ZITT ${ }^{2}$ \\ ${ }^{1}$ LMRS, Université de Rouen, Avenue de l'Université, BP 12, Technopôle du Madrillet, 76801 Saint- \\ Étienne-du-Rouvray, France.E-mail: jean-baptiste.bardet@univ-rouen.fr \\ ${ }^{2}$ LAMA UMR 8050, CNRS-Université-Paris-Est-Marne-La-Vallée, 5 boulevard Descartes, Cité Descartes, \\ Champs-sur-Marne, 77454 Marne-la-Vallée Cedex 2, France. \\ E-mail: natael.gozlan@u-pem.fr; pierre-andre.zitt@u-pem.fr \\ ${ }^{3}$ Laboratoire de Mathématiques et Physique Théorique (UMR CNRS 7350), Fédération Denis Poisson \\ (FR CNRS 2964), Université François-Rabelais, Parc de Grandmont, 37200 Tours, France. \\ E-mail: florent.malrieu@univ-tours.fr
}

The aim of this paper is to establish various functional inequalities for the convolution of a compactly supported measure and a standard Gaussian distribution on $\mathbb{R}^{d}$. We especially focus on getting good dependence of the constants on the dimension. We prove that the Poincaré inequality holds with a dimension-free bound. For the logarithmic Sobolev inequality, we improve the best known results (Zimmermann, JFA 2013) by getting a bound that grows linearly with the dimension. We also establish transport-entropy inequalities for various transport costs.

Keywords: logarithmic Sobolev inequality; Poincaré inequality; transport-entropy inequality

\section{Introduction}

Poincaré or logarithmic Sobolev inequalities have been extensively studied in the past decades to quantify long time behavior of Markov processes or investigate the concentration of measure property, which plays a key role for example in the topic of large random matrices.

We refer to $[3,4,13,19]$ for a comprehensive introduction to this subject. Let us briefly recall some well-known facts about these functional inequalities to motivate the present study.

A probability measure $v$ on $\mathbb{R}^{d}$ satisfies a Poincaré inequality with constant $C$ if, for any smooth function $f$ from $\mathbb{R}^{d}$ to $\mathbb{R}$,

$$
\int_{\mathbb{R}^{d}} f^{2} d \nu-\left(\int_{\mathbb{R}^{d}} f d \nu\right)^{2} \leq C \int_{\mathbb{R}^{d}}|\nabla f|^{2} d \nu .
$$

We denote by $C_{P}(v)$ the smallest constant such that this inequality holds. 
Similarly, $v$ satisfies a logarithmic Sobolev inequality with constant $C$ if, for any smooth function $f$ from $\mathbb{R}^{d}$ to $\mathbb{R}$,

$$
\int_{\mathbb{R}^{d}} f^{2} \log \left(f^{2}\right) d v-\left(\int_{\mathbb{R}^{d}} f^{2} d v\right) \log \left(\int_{\mathbb{R}^{d}} f^{2} d \nu\right) \leq C \int_{\mathbb{R}^{d}}|\nabla f|^{2} d v,
$$

and we denote by $C_{L S}(v)$ the smallest constant such that this inequality holds.

If $v$ is the Gaussian distribution $\mathcal{N}_{d}(x, \Gamma)$ on $\mathbb{R}^{d}$ with mean $x$ and covariance matrix $\Gamma$, then the values of these optimal constants are known:

$$
C_{P}(\nu)=\frac{1}{2} C_{L S}(\mu)=\max \operatorname{Spec}(\Gamma)
$$

The Bakry-Émery criterion ensures that if $v$ has the density $e^{-V}$ on $\mathbb{R}^{d}$ and $\operatorname{Hess}(V) \geq \rho I_{d}$ then

$$
C_{P}(v) \leq \frac{1}{\rho} \quad \text { and } \quad C_{L S}(\mu) \leq \frac{2}{\rho} .
$$

More generally, the inequality $2 C_{P}(v) \leq C_{L S}(v)$ always holds. These two functional inequalities do not hold if the support of $v$ is not connected - one can find a non constant function whose gradient is zero $v$-almost surely.

The present paper focuses on the case when the probability measure $v$ on $\mathbb{R}^{d}$ is given by the convolution $\mu \star \mathcal{N}_{d}\left(0, \delta^{2} I_{d}\right)$, where the support of $\mu$ is included in the centered ball of $\mathbb{R}^{d}$ with radius $R$. This question has been investigated recently in [21-23]; we present here several improvements and related questions.

Let us fix some notation first.

- $X$ and $Z$ are two independent random variables with respective distribution $\mu$ and $\mathcal{N}\left(0, I_{d}\right)$;

- $\gamma_{\delta}$ is the density of the Gaussian measure $\mathcal{N}_{d}\left(0, \delta^{2} I_{d}\right)$;

- $p$ is the density of the law $\mu \star \gamma_{\delta}$ of the random variable $S=X+\delta Z$;

- $C_{d}(\delta, R)$ is the supremum over all probability measures $\mu$ supported in the closed Euclidean ball $B_{d}(0, R)$ of the optimal constant in the logarithmic Sobolev inequality for $\mu \star \gamma_{\delta}$.

This notation is mainly consistent with [23], except that our $\delta$ is the standard deviation of the Gaussian rather than its variance, and we denote the dimension by $d$.

Zimmermann's results [22,23] may be summed up as follows.

Theorem 1.1 (Bounds on logarithmic Sobolev inequality constants [23]). The convolution of a compactly supported measure and a Gaussian measure satisfies a logarithmic Sobolev inequality. Moreover, there exist universal constants $\left(K_{i}\right)_{1 \leq i \leq 4}$ such that:

- In dimension 1 ,

$$
C_{1}(\delta, R) \leq K_{1} \frac{\delta^{3} R}{4 R^{2}+\delta^{2}} \exp \left(2 \frac{R^{2}}{\delta^{2}}\right)+K_{2}(\delta+2 R)^{2} .
$$


In particular in the low variance case $\delta \leq R$,

$$
C_{1}(\delta, R) \leq K_{3} \frac{\delta^{3}}{R} \exp \left(2 \frac{R^{2}}{\delta^{2}}\right) .
$$

- In dimension $d, C_{d}(\delta, R)$ is finite. In the low variance case $\delta \leq R$, it satisfies:

$$
C_{d}(\delta, R) \leq K_{4} R^{2} \exp \left(20 d+5 \frac{R^{2}}{\delta^{2}}\right) .
$$

The proofs in [23] rely on two main ideas. The one-dimensional case is treated by explicit computations on Hardy-like criteria taken from [6]. In higher dimension, the author applies the Lyapunov function approach of [7]. The constants $K_{i}$ are explicit but quite large (for example $K_{4}$ may be taken equal to 289). Let us also mention the alternate approach of [24] in dimension 1 by measure transportation, that unfortunately yields even worse constants. In the related note [21], Wang and Wang answer various related questions on functional inequalities for convolutions, and give many qualitative results under relaxed assumptions, both on the support of $X$ and on the distribution of the mollifier $Z$, but without exhibiting explicit constants.

We follow here the focus of [23] on quantitative estimates on the constants and their dependence on the dimension $d$. Our first result concerns the Poincaré inequality.

Theorem 1.2 (Dimension free Poincaré inequality). If $\mu$ is supported in the closed Euclidean ball $B_{d}(0, R)$, then $\mu \star \gamma_{\delta}$ satisfies a Poincaré inequality and

$$
C_{P}\left(\mu \star \gamma_{\delta}\right) \leq \delta^{2} \exp \left(4 \frac{R^{2}}{\delta^{2}}\right) .
$$

The next result is an improvement on the bounds of Theorem 1.1.

\section{Theorem 1.3 (Bounds on the logarithmic Sobolev constants).}

- In the large variance case $\delta>R$, the logarithmic Sobolev constants are bounded uniformly in the dimension:

$$
C_{d}(\delta, R) \leq \frac{\delta^{4}}{\delta^{2}-R^{2}}
$$

- In dimension 1, for any $\delta, R$,

$$
C_{1}(\delta, R) \leq 4 \delta^{2} \exp \left(\frac{8}{\pi} \frac{R^{2}}{\delta^{2}}\right) .
$$

- In the small variance case $\delta \leq R$, the logarithmic Sobolev constant admits the following dimension-dependent bound:

$$
C_{d}(\delta, R) \leq\left(K_{1} d+K_{2} \frac{R^{2}}{\delta^{2}}\right) R^{2} \exp \left(4 \frac{R^{2}}{\delta^{2}}\right),
$$

where $K_{1}, K_{2}$ are universal constants. 
The stronger bound in dimension 1 is obtained as a corollary of a bound that holds in any dimension (with a strong dependence on $d$ ). Its proof uses a trick by Miclo to apply the classical Holley-Stroock perturbation argument, and is much less technical than the ones in [23,24].

For the logarithmic Sobolev constant, the dependence in the dimension drops from exponential to linear: this enhancement would translate into weaker dependence assumptions in the applications to random matrices considered in [23].

In view of these results, it seems natural to conjecture as in [23] that $C_{d}(\delta, R)$ may admit a dimension free bound. Let us give some partial results in this direction.

The first is a dimension free bound for a transport-entropy inequality. We recall that if $k$ : $\mathbb{R}^{d} \times \mathbb{R}^{d} \rightarrow \mathbb{R}^{+}$is a cost function, then the optimal transport cost related to this $k$, is defined, for all probability measures $v_{1}$ and $v_{2}$, by

$$
\mathcal{T}_{k}\left(v_{1}, v_{2}\right)=\inf _{\pi} \int k(x, y) d \pi(x, y),
$$

where the infimum is taken over the set of all couplings $\pi$ between $\nu_{1}$ and $\nu_{2}$. Let $\mathcal{T}_{2,4}$ and $\mathcal{T}_{2}$ denote the transportation costs associated to $(x, y) \mapsto\|x-y\|_{4}^{2}$ and $(x, y) \mapsto|x-y|^{2}$ (here and in the whole paper, $|\cdot|$ denotes the Euclidean norm).

Theorem 1.4 (Transportation-entropy inequality). Let $\mu$ be a probability measure on $\mathbb{R}^{d}$ supported in $B_{d}(0, R)$. The probability $\mu \star \gamma_{\delta}$ satisfies the following transport-entropy inequalities: for any probability measure $v$ on $\mathbb{R}^{d}$,

$$
\begin{aligned}
\mathcal{T}_{2,4}\left(\nu, \mu \star \gamma_{\delta}\right) & \leq C(R, \delta) H\left(\nu \mid \mu \star \gamma_{\delta}\right), \\
\mathcal{T}_{2}\left(\nu, \mu \star \gamma_{\delta}\right) & \leq \sqrt{d} C(R, \delta) H\left(\nu \mid \mu \star \gamma_{\delta}\right),
\end{aligned}
$$

where $C(R, \delta)=c^{\prime} \delta^{2}\left(1+\frac{R^{2}}{\delta^{2}}\right) \exp \left(\frac{4 R^{2}}{\delta^{2}}\right)$ for some universal constant $c^{\prime}$.

Remark 1. We obtain here a transportation-entropy inequality with a constant that scales like $\sqrt{d}$ : this is an improvement over the linear scaling that would follow from applying Otto-Villani's theorem (see $[5,18]$ ) to deduce the $\mathcal{T}_{2}$ transport inequality from the logarithmic Sobolev inequality (1). The price to pay for this improvement is that the proof of Theorem 1.4 relies on rather technical estimates on an intricate cost function (see Section 3.4).

Finally, we are able to get bounds on the logarithmic Sobolev constant in several restricted cases.

\section{Theorem 1.5 (Partial results).}

- The quantity $C_{d}(\delta, R)$ may be bounded only in terms of $\delta$ and $R$ in the region $\delta>R / \sqrt{2}$.

- If $\mu$ is radially symmetric, then

$$
C_{L S}\left(\mu \star \gamma_{\delta}\right) \leq 4 \delta^{2} \exp \left(\frac{8}{\pi} \frac{R^{2}}{\delta^{2}}\right) .
$$


- If $\mu=\sum_{i=1}^{N} p_{i} \delta_{x_{i}}$ is a discrete probability measure on $N \geq 3$ points, with minimal weight $p_{\star}=\min \left(p_{i}\right)$, then

$$
C_{L S}\left(\mu \star \gamma_{\delta}\right) \leq \delta^{2}+3 \log \left(1 / p_{\star}\right) \delta^{2} \exp \left(4 \frac{R^{2}}{\delta^{2}}\right) .
$$

- The logarithmic Sobolev inequality restricted to log-convex functions holds with a constant that does not depend on the dimension.

To prove or disprove the conjecture, one is tempted to guess the measure $\mu$ that leads to the worst logarithmic Sobolev constant. A natural candidate, proposed in [23], Example 21, is the two-point measure $1 / 2\left(\delta_{R e_{1}}+\delta_{-R e_{1}}\right)$ (where $e_{1}$ denotes the first basis vector). Note that this candidate is easily seen to satisfy a logarithmic Sobolev inequality with a bounded constant, either by the bound on discrete measures or by a simple tensorization argument of a one-dimensional convolution with a $(d-1)$-dimensional Gaussian law. To build a counterexample, one would have to consider measures with a number of points that grows with the dimension.

Remark 2 (Discrete measures). Since the support of a discrete measure on $N$ points is included in an $(N-1)$-dimensional affine space, the same tensorization argument of an $(N-1)$ dimensional convolution with a $(d-N+1)$-dimensional Gaussian law shows that $C_{L S}\left(\mu \star \gamma_{\delta}\right)$ is also bounded by $\left(K_{1} N+K_{2} \frac{R^{2}}{\delta^{2}}\right) R^{2} \exp \left(4 R^{2} / \delta^{2}\right)$. This gives a better bound than Theorem 1.5 when $N$ is fixed and the minimal weight $p_{\star}$ vanishes.

\section{Outline of the paper}

The paper is organized as follows. In Section 2, we use the perturbation idea of Holley-Stroock, by rewriting the potential of $\mu \star \gamma_{\delta}$ as a sum of a convex function and a bounded perturbation, proving the first two items of Theorem 1.3. In Section 3, viewing $\mu \star \gamma_{\delta}$ as a mixture of Gaussian measures we prove the Poincaré and transportation inequalities (Theorems 1.2 and 1.4) and establish the bound for discrete measures (third item of Theorem 1.5). Theorem 1.2 yields the final bound on logarithmic Sobolev constants (the third item in Theorem 1.3) as an easy corollary. The various remaining results in Theorem 1.5 are proved in Section 4.

\section{Perturbation arguments}

\subsection{Large variance}

The density $p$ of $\mu \star \gamma_{\delta}$ is given explicitly by:

$$
p(z)=\int_{\mathbb{R}^{d}} \frac{1}{\left(2 \pi \delta^{2}\right)^{d / 2}} \exp \left(-\frac{|z-x|^{2}}{2 \delta^{2}}\right) \mu(d x)=\frac{1}{\left(2 \pi \delta^{2}\right)^{d / 2}} \exp \left(-\left(\frac{|z|^{2}}{2 \delta^{2}}+W_{\delta}(z)\right)\right),
$$


where

$$
\begin{aligned}
W_{\delta}(z) & =-\log \int_{\mathbb{R}^{d}} \exp \left(\frac{z \cdot x}{\delta^{2}}-\frac{|x|^{2}}{2 \delta^{2}}\right) \mu(d x) \\
& =-\log \int_{\mathbb{R}^{d}} \exp \left(\frac{z \cdot x}{\delta^{2}}\right) \nu(d x)-\log C_{v}
\end{aligned}
$$

for $C_{v}=\int_{\mathbb{R}^{d}} \exp \left(-|x|^{2} /\left(2 \delta^{2}\right)\right) \mu(d x)$ and $\nu(d x)=C_{v}^{-1} \exp \left(-|x|^{2} /\left(2 \delta^{2}\right)\right) \mu(d x)$. Let us compute the Hessian of $\left(-W_{\delta}\right)$ :

$$
\partial_{z_{i}}\left(-W_{\delta}\right)(z)=\frac{1}{\delta^{2}} \mathbb{E}\left(\tilde{X}_{i}\right) \quad \text { and } \quad \partial_{z_{i} z_{j}}^{2}\left(-W_{\delta}\right)(z)=\frac{1}{\delta^{4}} \operatorname{Cov}\left(\tilde{X}_{i}, \tilde{X}_{j}\right),
$$

where the distribution of $\tilde{X}$ is proportional to $\exp (z \cdot x) d v$. Therefore, for any unit vector $v$,

$$
0 \leq \operatorname{Hess}\left(-W_{\delta}\right) v \cdot v \leq \frac{1}{\delta^{4}} \operatorname{Var}(v \cdot \tilde{X}) .
$$

Since $v \cdot \tilde{X}$ lives in $[-R, R]$, its variance is bounded by $R^{2}$, so

$$
\operatorname{Hess}(-\log (p)) \geq\left(\frac{1}{\delta^{2}}-\frac{R^{2}}{\delta^{4}}\right) I_{d}
$$

Remark 3. This bound is slightly better than the one given in [23] where the variance of $v \cdot \tilde{X}$ is bounded by $2 R^{2}$.

In particular, if $\delta>R, p$ is log-concave and the Bakry-Émery criterion yields:

$$
C_{L S}\left(\mu \star \gamma_{\delta}\right) \leq \frac{\delta^{4}}{\delta^{2}-R^{2}}
$$

This proves the first item in Theorem 1.3.

\subsection{A perturbation argument}

It turns out we can get a (dimension dependent) bound on the logarithmic Sobolev constant with a very short proof, using the following trick to decompose the logarithm of the density $p$ as a sum of a convex function and a bounded perturbation.

Let $a_{d}=\mathbb{E}[|Z|]$ be the expected value of the norm of a standard Gaussian random variable $Z$ in dimension $d$. Note that $a_{d}$ has an explicit expression (we will use below that $a_{1}=\sqrt{2 / \pi}$ ) and is in any case smaller than $\sqrt{d}$.

Lemma 2.1 (Miclo's trick [14,19]). Suppose the function $W: \mathbb{R}^{d} \rightarrow \mathbb{R}$ may be written as $W=$ $W_{c}+W_{l}$, where $\operatorname{Hess}\left(W_{c}\right) \geq \rho I_{d}$, and $W_{l}$ is l-Lipschitz with respect to the Euclidean distance. 
Then for any $\sigma>0$, one can write $W$ as a sum $U_{c}+U_{b}$, where $\operatorname{Hess}\left(U_{c}\right) \geq\left(\rho-\frac{l a_{1}}{\sigma}\right) I_{d}$ and $U_{b}$ is bounded by $l \sigma a_{d}$.

In particular, the measure $Z_{W}^{-1} \exp (-W)$ satisfies a logarithmic Sobolev inequality and

$$
C_{L S}\left(Z_{W}^{-1} \exp (-W)\right) \leq \frac{4}{\rho} \exp \left(\frac{4}{\rho} l^{2} a_{1} a_{d}\right)
$$

By way of comparison, it is known (see [1,2]) that if $\mu_{0}=\exp \left(-V_{0}\right) d x$ satisfies a logarithmic Sobolev inequality, then $v=\exp (-V) d x$ satisfies a defective logarithmic Sobolev inequality, as soon as the gradient $\nabla\left(V-V_{0}\right)$ satisfies some exponential integrability condition. This defective inequality can be used together with the Poincaré inequality to obtain the logarithmic Sobolev inequality. This strategy is used in [21] (see in particular Lemma 2.3 of [21] for a precise statement of the perturbation result). It is more general, since it only supposes a logarithmic Sobolev inequality for the unperturbed measure, and replaces a boundedness assumption by an integrability condition. The trade-off is that the constants are not explicit.

Since the statement of Lemma 2.1 in $[14,19]$ contains a typo in the convexity bound, let us provide a detailed proof.

Proof of Lemma 2.1. Let $\sigma>0$ and $U_{\sigma}$ be the following regularized version of $W_{l}: U_{\sigma}(x)=$ $\mathbb{E}\left[W_{l}(x+\sigma Z)\right]$, where $Z$ is a standard $d$-dimensional Gaussian random variable. Let $U_{c}=$ $W_{c}+U_{\sigma}$ and $U_{b}=W_{l}-U_{\sigma}$. Since $W_{l}$ is $l$-Lipschitz,

$$
\left|U_{b}(x)\right|=\left|\mathbb{E}\left[W_{l}(x)-W_{l}(x+\sigma Z)\right]\right| \leq l \sigma \mathbb{E}[|Z|] \leq l \sigma a_{d} .
$$

Therefore, $U_{b}$ is bounded.

We now turn to the convexity bound. It is enough to prove that, for any unit vector $v$ in $\mathbb{R}^{d}$, Hess $U_{\sigma} v \cdot v \leq \frac{l c_{1}}{\sigma}$. First, we compute the derivatives of $U_{\sigma}$ :

$$
\begin{aligned}
\partial_{i} U_{\sigma}(x) & =\left(2 \pi \sigma^{2}\right)^{-d / 2} \frac{1}{\sigma^{2}} \int_{\mathbb{R}^{d}} W_{l}(y)\left(y_{i}-x_{i}\right) \exp \left(-\frac{|x-y|^{2}}{2 \sigma^{2}}\right) d y \\
& =\left(2 \pi \sigma^{2}\right)^{-d / 2} \frac{1}{\sigma^{2}} \int_{\mathbb{R}^{d}} W_{l}(x+z) z_{i} \exp \left(-\frac{|z|^{2}}{2 \sigma^{2}}\right) d z, \\
\partial_{i j} U_{\sigma}(x) & =\left(2 \pi \sigma^{2}\right)^{-d / 2} \frac{1}{\sigma^{2}} \int_{\mathbb{R}^{d}} \partial_{j} W_{l}(x+z) z_{i} \exp \left(-\frac{|z|^{2}}{2 \sigma^{2}}\right) d z .
\end{aligned}
$$

Now,

$$
\text { Hess } U_{\sigma} v \cdot v=\left(2 \pi \sigma^{2}\right)^{-d / 2} \frac{1}{\sigma^{2}} \int_{\mathbb{R}^{d}}\left(v \cdot \nabla W_{l}(x+z)\right)(v \cdot z) \exp \left(-\frac{|z|^{2}}{2 \sigma^{2}}\right) d z \text {. }
$$

Since $W_{l}$ is $l$-Lipschitz,

$$
\left|\operatorname{Hess} U_{\sigma} v \cdot v\right| \leq\left(2 \pi \sigma^{2}\right)^{-d / 2} \frac{l}{\sigma^{2}} \int_{\mathbb{R}^{d}}|v \cdot z| \exp \left(-\frac{|z|^{2}}{2 \sigma^{2}}\right) d z .
$$


By rotation invariance of the standard Gaussian distribution, we get

$$
\left|\operatorname{Hess} U_{\sigma} v \cdot v\right| \leq \frac{l}{\sigma^{2}} \mathbb{E}\left[\sigma\left|Z_{1}\right|\right] \leq \frac{l a_{1}}{\sigma}
$$

This implies that $\operatorname{Hess}\left(W_{c}+U_{\sigma}\right) \geq\left(\rho-\frac{l a_{1}}{\sigma}\right) I_{d}$, as claimed.

The final claim is a direct consequence of the obtained decomposition with $\sigma=2 l a_{1} / \rho$, the Holley-Stroock perturbation lemma and the Bakry-Émery criterion (see [19]).

Let us now use this lemma to prove the one-dimensional bound in Theorem 1.3. Write $-\log (p)$ as

$$
-\log (p(z))=\left(\frac{|z|^{2}}{2 \delta^{2}}+\frac{d}{2} \log \left(2 \pi \delta^{2}\right)\right)+W_{\delta}(z) .
$$

The first term is $\delta^{-2}$-convex. Since

$$
\nabla W_{\delta}(z)=-\frac{1}{\delta^{2}} \frac{\int_{\mathbb{R}^{d}} x \exp \left(\frac{z \cdot x}{\delta^{2}}\right) v(d x)}{\int_{\mathbb{R}^{d}} \exp \left(\frac{z \cdot x}{\delta^{2}}\right) v(d x)}
$$

and $v\left(B_{d}(0, R)\right)=1, W_{\delta}$ is $R / \delta^{2}$-Lipschitz on $\mathbb{R}^{d}$. Lemma 2.1 then yields

$$
C_{L S}\left(\mu \star \gamma_{\delta}\right) \leq 4 \delta^{2} \exp \left(4 a_{1} a_{d} R^{2} \delta^{-2}\right) .
$$

This gives a first dimension dependent bound that is not comparable to the one from Theorem 1.1. In dimension 1 , since $a_{1}=\sqrt{2 / \pi}$, we get the bound claimed in the second item of Theorem 1.3.

\section{Mixture arguments}

\subsection{Poincaré inequality}

In this section, we denote by $\gamma_{x, \delta}$ the distribution $\mathcal{N}_{d}\left(x, \delta^{2} I_{d}\right)$. Recall that $\mu \star \gamma_{\delta}=$ $\int_{\mathbb{R}^{d}} \gamma_{x, \delta} d \mu(x)$. The variance of a function $f$ under the mixture $\mu \star \gamma_{\delta}$ can be classically decomposed as

$$
\begin{aligned}
\operatorname{Var}_{\mu \star \gamma_{\delta}}(f) & =\int_{\mathbb{R}^{d}} \operatorname{Var}_{\gamma_{x, \delta}}(f) d \mu(x)+\operatorname{Var}_{\mu}\left(x \mapsto \int f d \gamma_{x, \delta}\right) \\
& =A+B .
\end{aligned}
$$

Since $\gamma_{x, \delta}$ satisfies the Poincaré inequality with constant $\delta^{2}$, the first term $A$ is bounded by

$$
\delta^{2} \int_{\mathbb{R}^{d}} \int_{\mathbb{R}^{d}}|\nabla f|^{2} d \gamma_{x, \delta} d \mu(x)=\delta^{2} \int_{\mathbb{R}^{d}}|\nabla f|^{2} d\left(\mu \star \gamma_{\delta}\right) .
$$


For the second term, $B$ let $g: x \mapsto \int_{\mathbb{R}^{d}} f d \gamma_{x, \delta}$. Duplicating variables yields

$$
B=\frac{1}{2} \iint_{\mathbb{R}^{d} \times \mathbb{R}^{d}}(g(x)-g(y))^{2} d \mu(x) d \mu(y) .
$$

Now

$$
\begin{aligned}
(g(x)-g(y))^{2} & =\left(\int_{\mathbb{R}^{d}} f d \gamma_{x, \delta}-\int_{\mathbb{R}^{d}} f d \gamma_{y, \delta}\right)^{2} \\
& =\left(\int_{\mathbb{R}^{d}} f\left(1-\frac{d \gamma_{y, \delta}}{d \gamma_{x, \delta}}\right) d \gamma_{x, \delta}\right)^{2} \\
& =\left(\operatorname{Cov}_{\gamma_{x, \delta}}\left(f,\left(1-\frac{d \gamma_{y, \delta}}{d \gamma_{x, \delta}}\right)\right)\right)^{2} \\
& \leq \operatorname{Var}_{\gamma_{x, \delta}}(f) \operatorname{Var}_{\gamma_{x, \delta}}\left(1-\frac{d \gamma_{y, \delta}}{d \gamma_{x, \delta}}\right)
\end{aligned}
$$

by Cauchy-Schwarz inequality. For the first factor, we reapply the Poincare inequality for the Gaussian measure $\gamma_{x, \delta}$. The second factor is the $\chi^{2}$ divergence between the Gaussian distributions $\gamma_{x, \delta}$ and $\gamma_{y, \delta}$. An easy computation shows that this divergence is $\left(\exp \left(|x-y|^{2} / \delta^{2}\right)-1\right)$; since $|x-y|$ is bounded by $2 R$, we get

$$
(g(x)-g(y))^{2} \leq \delta^{2}\left(\exp \left(4 R^{2} / \delta^{2}\right)-1\right) \int_{\mathbb{R}^{d}}|\nabla f|^{2} d \gamma_{x, \delta} .
$$

Reintegrating with respect to $\mu$ yields

$$
B \leq \delta^{2}\left(\exp \left(4 R^{2} / \delta^{2}\right)-1\right) \int_{\mathbb{R}^{d}}|\nabla f|^{2} d\left(\mu \star \gamma_{\delta}\right),
$$

so that the measure $\mu \star \gamma_{\delta}$ satisfies a Poincaré inequality with a constant

$$
C_{P}\left(\mu \star \gamma_{\delta}\right) \leq \delta^{2} \exp \left(4 R^{2} / \delta^{2}\right) .
$$

\subsection{A mild dependence on $\boldsymbol{d}$ for logarithmic Sobolev constants via Lyapunov functions}

The proof of the logarithmic Sobolev inequality in dimension greater than 1 in [23] is based on a criterion from [7]. This criterion uses a Lyapunov function approach to prove a so-called defective logarithmic Sobolev inequality, which can then be strengthened using the Poincaré inequality. In [23], this Poincaré inequality is itself obtained by Lyapunov criteria, with constants depending exponentially on the dimension. Simply plugging our dimension-free Poincaré inequality in the argument of [7] gives a much better bound.

Let us first recall the criterion, in the form used in [23], where the constants are explicitly written. 
Theorem 3.1 (Logarithmic Sobolev inequality via Lyapunov functions [7]). Suppose that $V$ satisfies

$$
\operatorname{Hess}(V) \geq-K I_{d}
$$

with $K \geq 0$, and there exists a "Lyapunov function", that is, a function $W \geq 1$ such that

$$
\Delta W-\langle\nabla V, \nabla W\rangle \leq\left(b-c|x|^{2}\right) W
$$

for some positive constants $b, c$.

Suppose that $v=Z_{V}^{-1} \exp (-V) d x$ satisfies a Poincaré inequality with constant $C_{P}(v)$. Let $A$ and $B$ be defined by

$$
\begin{aligned}
& A=\frac{2}{c}\left(\varepsilon^{-1}+K / 2\right)+\varepsilon \\
& B=\frac{2}{c}\left(\varepsilon^{-1}+K / 2\right)\left(b+c \int_{\mathbb{R}^{d}}|x|^{2} d \nu(x)\right) .
\end{aligned}
$$

Then $v$ satisfies a logarithmic Sobolev inequality and $C_{L S}(v) \leq A+(B+2) C_{P}(v)$.

Zimmermann proves in [23] that (2) holds with $b=d /\left(8 \delta^{2}\right)+R^{2} /\left(32 \delta^{4}\right)$ and $c=\frac{1}{64 \delta^{4}}$ for the function $W(x)=\exp \left(\frac{1}{64 \delta^{4}}\right)$. Using the bound $K \leq R^{2} / \delta^{4}$ and choosing $\varepsilon=2 / K$, this proves that, for $\delta \leq R$, thanks to the bound on the Poincaré constant,

$$
C_{L S}\left(\mu \star \gamma_{\delta}\right) \leq\left(K_{1} d+K_{2} \frac{R^{2}}{\delta^{2}}\right) R^{2} \exp \left(4 \frac{R^{2}}{\delta^{2}}\right)
$$

for some universal constants $K_{1}, K_{2}$, which is the general bound announced in Theorem 1.3.

\subsection{A bound for discrete measures}

Suppose in this section that $\mu$ is a probability measure on $N$ points in $B_{d}(0, R)$ :

$$
\mu=\sum_{i=1}^{N} p_{i} \delta_{x_{i}}
$$

The distribution of $S=X+Z_{\delta}$ is a mixture of $N$ Gaussian laws with respective means $x_{i}$ and common covariance matrix $\delta^{2} I_{d}$. Poincaré and logarithmic Sobolev inequalities for mixtures of two measures have been studied by Chafaï and Malrieu in [8]; Schlichting and Menz [16,20] have used and generalized their results to prove Eyring-Kramers formulæ. The decomposition of the variance used in Section 3.1 has the following analogue for entropies:

$$
\operatorname{Ent}_{\mu \star \gamma_{\delta}}\left(f^{2}\right)=\int_{\mathbb{R}^{d}} \operatorname{Ent}_{\gamma_{x, \delta}}\left(f^{2}\right) d \mu(x)+\operatorname{Ent}_{\mu}\left(x \mapsto \int_{\mathbb{R}^{d}} f^{2} d \gamma_{x, \delta}\right) .
$$


To bound the second term, we use the following result, that is essentially a consequence of the discrete logarithmic Sobolev inequality for the complete graph proved by Diaconis and SaloffCoste in [9].

Theorem 3.2 (Upper bound for the entropy when $\mu$ is discrete [20]). Let $\mu=\sum_{i=1}^{N} p_{i} \mu_{i}$ be a finite mixture of measures. Let $p_{\star}=\min _{1 \leq i \leq N} p_{i}$. Then for any $f$,

$$
\operatorname{Ent}_{\mu}\left(i \mapsto \int_{\mathbb{R}^{d}} f^{2} d \mu_{i}\right) \leq \frac{1}{\Lambda\left(p_{\star}, 1-p_{\star}\right)}\left(\sum_{i=1}^{N} p_{i} \operatorname{Var}_{\mu_{i}}(f)+\operatorname{Var}_{\mu}\left(i \mapsto \int_{\mathbb{R}^{d}} f d \mu_{i}\right)\right)
$$

where $\Lambda(p, q)=(p-q) /(\log p-\log q)$.

Remark 4. For discrete chains, much more general results are available for various definitions of the discrete gradient appearing in the energy, see, for example, [12]. Unfortunately, we were not able to relate these energies to the continuous one $\int|\nabla f|^{2} d \mu$.

Proof of Theorem 3.2. This follows from Corollary 2.18 of [20], using the result from [9] instead of the alternate Lemma 2.13 of [20].

Coming back to the decomposition (3), we can use the Gaussian logarithmic Sobolev inequality on the first term and Theorem 3.2 on the second term to get:

$$
\begin{aligned}
\operatorname{Ent}_{\mu \star \gamma \delta}\left(f^{2}\right) \leq & 2 \delta^{2} \int_{\mathbb{R}^{d}}|\nabla f|^{2} d \mu(x) \\
& +\frac{1}{\Lambda\left(p_{\star}, 1-p_{\star}\right)}\left(\sum_{i=1}^{N} p_{i} \operatorname{Var}_{\gamma_{\delta, x_{i}}}(f)+\operatorname{Var}_{\mu}\left(i \mapsto \int_{\mathbb{R}^{d}} f d \gamma_{\delta, x_{i}}\right)\right) .
\end{aligned}
$$

The last bracket is the variance $\operatorname{Var}_{\mu \star \gamma_{\delta}}(f)$, which is bounded thanks to the Poincare inequality. Since $\frac{1}{\Lambda(p, 1-p)} \leq \frac{\log (1 / p)}{1-2 p}$ and $p_{\star} \leq(1 / N) \leq 1 / 3$, we finally get

$$
C_{L S}\left(\mu \star \gamma_{\delta}\right) \leq 2 \delta^{2}+3 \log \left(1 / p_{\star}\right) \delta^{2} \exp \left(4 R^{2} / \delta^{2}\right) .
$$

\subsection{Dimension free transport-entropy inequality for the $\ell^{4}$ norm}

We now adapt the arguments of Section 3.1 to prove that the measure $\mu \star \gamma_{\delta}$ satisfies a transportentropy inequality with a constant depending only on $R$ and $\delta$. It is more convenient in this section to state and prove all intermediate results for $\delta=1$. In the final result, we come back to the general case by an immediate scaling argument.

The first step is to establish a weighted version of the Poincaré inequality. 
Lemma 3.3 (Weighted Poincaré inequality for Gaussian measures). For all $x \in \mathbb{R}^{d}$, the Gaussian measure $\gamma_{x, 1}$ satisfies the following weighted Poincaré inequality: for all $\mathcal{C}^{1}$ function $f$,

$$
\operatorname{Var}_{\gamma_{x, 1}}(f) \leq c\left(1+|x|^{2}\right) \int_{\mathbb{R}^{d}} \sum_{i=1}^{d} \frac{1}{1+u_{i}^{2}}\left(\partial_{i} f(u)\right)^{2} d \gamma_{x, 1}(u),
$$

where $c$ is a positive universal constant.

Proof. Let us first establish the result for the standard Gaussian distribution $\gamma=\mathcal{N}(0,1)$ in dimension $d=1$. According to the well known Muckenhoupt criterion for Hardy type inequalities (see, e.g., Theorem 6.2.1 in [3]), the inequality

$$
\int_{0}^{\infty}(f(u)-f(0))^{2} d \gamma(u) \leq c \int_{0}^{\infty} \frac{1}{1+u^{2}} f^{\prime}(u)^{2} d \gamma(u)
$$

holds for all $\mathcal{C}^{1}$ function $f:[0, \infty) \rightarrow \mathbb{R}$, with the constant

$$
c=\sup _{y \geq 0} \int_{y}^{\infty} e^{-u^{2} / 2} d u \int_{0}^{y}\left(1+u^{2}\right) e^{u^{2} / 2} d u<\infty .
$$

Similarly, for any $\mathcal{C}^{1}$ function $f$ on $(-\infty, 0]$, it holds

$$
\int_{-\infty}^{0}(f(u)-f(0))^{2} d \gamma(u) \leq c \int_{-\infty}^{0} \frac{1}{1+u^{2}} f^{\prime}(u)^{2} d \gamma(u) .
$$

Therefore, if $f$ is now $\mathcal{C}^{1}$ function on $\mathbb{R}$, one has

$$
\operatorname{Var}_{\gamma}(f) \leq \int_{\mathbb{R}}(f(u)-f(0))^{2} d \gamma(u) \leq c \int_{\mathbb{R}} \frac{1}{1+u^{2}} f^{\prime}(u)^{2} d \gamma(u) .
$$

Applying this inequality to $f(u)=g(x+u), u \in \mathbb{R}$, yields

$$
\operatorname{Var}_{\gamma_{x, 1}}(g) \leq c \int_{\mathbb{R}} \frac{1}{1+(v-x)^{2}} g^{\prime}(v)^{2} d \gamma_{x, 1}(v) .
$$

Since $1+v^{2} \leq 1+2(v-x)^{2}+2 x^{2} \leq 2\left(1+x^{2}\right)\left(1+(x-v)^{2}\right)$, the claim holds for the Gaussian measure $\gamma_{x, 1}$ in dimension 1 .

To prove the general case, just remark that, for any $x \in \mathbb{R}^{d}, \gamma_{x, 1}$ is the product of the (one dimensional) measures $\gamma_{1, x_{i}}$. The classical tensorization property for Poincaré-type inequalities yields

$$
\operatorname{Var}_{\gamma_{x, 1}}(g) \leq 2 c \max _{i}\left(1+x_{i}^{2}\right) \int_{\mathbb{R}} \sum_{i=1}^{d} \frac{1}{1+v_{i}^{2}}\left(\partial_{i} g(v)\right)^{2} d \gamma_{x, 1}(v),
$$

which completes the proof. 
This result extends to mixture of Gaussian measures.

Proposition 3.4 (Weighted Poincaré inequality for $\mu \star \gamma_{1}$ ). Let $\mu$ be a probability measure on $\mathbb{R}^{d}$ supported in $B_{d}(0, R)$. The probability $\mu \star \gamma_{\delta}$ satisfies the following weighted Poincaré inequality: for all $\mathcal{C}^{1}$ function $f$ on $\mathbb{R}^{d}$,

$$
\operatorname{Var}_{\mu \star \gamma_{1}}(f) \leq C(R) \int_{\mathbb{R}^{d}} \sum_{i=1}^{d} \frac{1}{1+u_{i}^{2}}\left(\partial_{i} f(u)\right)^{2} d\left(\mu \star \gamma_{1}\right)(u),
$$

with $C(R)=c\left(1+R^{2}\right) e^{4 R^{2}}$ for some universal constant $c$.

Proof. According to Lemma 3.3, for all $x \in \mathbb{R}^{d}$ such that $|x| \leq R$, it holds

$$
\operatorname{Var}_{\gamma_{x, 1}}(f) \leq c\left(1+R^{2}\right) \int_{\mathbb{R}} \sum_{i=1}^{d} \frac{1}{1+u_{i}^{2}}\left(\partial_{i} f(u)\right)^{2} d \gamma_{x, 1}(u)
$$

for all $\mathcal{C}^{1}$ function $f$ on $\mathbb{R}^{d}$. Inserting these weighted Poincaré inequalities into the proof given in Section 3.1 immediately yields the desired bound.

We now arrive at a first transportation-entropy inequality.

Theorem 3.5. Let $\mu$ be a probability measure on $\mathbb{R}^{d}$ having its support in $B_{d}(0, R)$. The probability $\mu \star \gamma_{1}$ satisfies the following transport-entropy inequality: for any probability measure $v$ on $\mathbb{R}^{d}$,

$$
\mathcal{T}_{k}\left(\nu, \mu \star \gamma_{1}\right) \leq c^{\prime}\left(1+R^{2}\right) \exp \left(4 R^{2}\right) H\left(\nu \mid \mu \star \gamma_{1}\right),
$$

where $c^{\prime}$ is a universal constant and $\mathcal{T}_{k}$ is the optimal transport cost related to the cost function

$$
k(x, y)=\min \left(|x-y|^{2} ;|x-y|\right)+\min \left(\|x-y\|_{4}^{4},\|x-y\|_{4}^{2}\right), \quad \forall x, y \in \mathbb{R}^{d} .
$$

Before proving this result, let us show how to deduce Theorem 1.4 as a corollary. The Euclidean and $\ell^{4}$ norms on $\mathbb{R}^{d}$ satisfy:

$$
\forall z \in \mathbb{R}^{d}, \quad\|z\|_{4} \leq|z| \leq d^{1 / 4}\|z\|_{4} .
$$

This gives the following lower bound on the cost $k$ :

$$
\begin{aligned}
k(x, y) & =\min \left(|x-y|^{2} ;|x-y|\right)+\min \left(\|x-y\|_{4}^{4},\|x-y\|_{4}^{2}\right) \\
& \geq \min \left(\|x-y\|_{4}^{2} ;\|x-y\|_{4}\right)+\min \left(\|x-y\|_{4}^{4},\|x-y\|_{4}^{2}\right) \\
& \geq\|x-y\|_{4}^{2} .
\end{aligned}
$$

By Theorem 3.5 we get

$$
\mathcal{T}_{2,4}\left(\nu, \mu \star \gamma_{1}\right) \leq \mathcal{T}_{k}\left(\nu, \mu \star \gamma_{1}\right) \leq c^{\prime}\left(1+R^{2}\right) \exp \left(4 R^{2}\right) H\left(\nu \mid \mu \star \gamma_{1}\right)
$$


where we recall that $\mathcal{T}_{2,4}$ is the transportation cost associated to $(x, y) \mapsto\|x-y\|^{4}$. The inequality for a general $\delta$ follows by a simple scaling argument. The inequality for the Euclidean cost $\mathcal{T}_{2}$ is proved in the same way, by bounding $k(x, y)$ from below by $d^{-1 / 2}|x-y|^{2}$. This concludes the proof of Theorem 1.4.

Proof of Theorem 3.5. We proceed in two steps.

Step 1. A transport-entropy inequality with an intricate cost. Let us define three functions $\alpha$, $\omega$ and $T$ by

$$
\begin{aligned}
\forall u \in \mathbb{R}, & \omega(u)=\operatorname{sign}(u)\left(|u|+\frac{u^{2}}{2}\right) ; \\
\forall u \in \mathbb{R}, & \alpha(u)=\min \left(u^{2} ;|u|\right) ; \\
\forall x \in \mathbb{R}^{d}, & T(x)=\left(\omega\left(x_{1}\right), \ldots, \omega\left(x_{d}\right)\right) .
\end{aligned}
$$

According to Theorem 4.6 in [10], the weighted Poincaré inequality (4) implies (and is actually equivalent to) the following transport cost inequality: for all probability measure $v$ on $\mathbb{R}^{d}$,

$$
\mathcal{T}_{\tilde{k}}\left(\nu, \mu \star \gamma_{1}\right) \leq H\left(\nu \mid \mu \star \gamma_{\delta}\right),
$$

where the cost function $\tilde{k}$ is defined by

$$
\tilde{k}(x, y)=\alpha\left(\frac{1}{D}|T(x)-T(y)|\right), \quad \forall x, y \in \mathbb{R}^{d}
$$

and where $D=c^{\prime \prime} \sqrt{C(R)}$ for some universal constant $c^{\prime \prime}$.

For the sake of completeness, let us give the short proof of the implication we need. Let us begin by showing that the measure $\tilde{\mu}:=T_{\#}\left(\mu \star \gamma_{1}\right)$ satisfies the usual Poincaré inequality with the constant $2 C(R)$. Indeed, if $f$ is a $\mathcal{C}^{1}$ function, applying the weighted Poincare inequality (4) to $g=f \circ T$ and using the elementary bound $\left(\omega^{\prime}(v)\right)^{2} \leq 2\left(1+v^{2}\right)$ yields:

$$
\begin{aligned}
\operatorname{Var}_{\tilde{\mu}}(f) & \leq C(R) \int \sum_{i=1}^{d} \frac{1}{1+v_{i}^{2}} \omega^{\prime}\left(v_{i}\right)^{2}\left(\partial_{i} f\right)^{2}(T(v)) d\left(\mu \star \gamma_{1}\right)(v) \\
& \leq 2 C(R) \int|\nabla f|^{2}(u) d \tilde{\mu}(u) .
\end{aligned}
$$

According to a well-known result by Bobkov, Gentil and Ledoux [5], Corollary 5.1 showing the equivalence between the Poincaré inequality and a transport inequality involving a quadraticlinear cost, the probability $\tilde{\mu}$ satisfies the following: for any probability measure $v$ on $\mathbb{R}^{d}$,

$$
\mathcal{T}_{\rho}(\nu, \tilde{\mu}) \leq H(\nu \mid \tilde{\mu}),
$$

where the cost function $\rho: \mathbb{R}^{d} \times \mathbb{R}^{d} \rightarrow \mathbb{R}^{+}$is defined by

$$
\rho(x, y)=\alpha\left(\frac{1}{D}|x-y|\right), \quad x, y \in \mathbb{R}^{d},
$$


where $D=c^{\prime \prime} \sqrt{C(R)}$ for some universal constant $c^{\prime \prime}$. Let $v$ be a probability measure on $\mathbb{R}^{d}$ and let $(\tilde{X}, \tilde{Y})$ be an optimal coupling between $\tilde{v}:=T_{\#} v$ and $\tilde{\mu}$ (for the transport cost $\mathcal{T}_{\rho}$ ) and denote by $X=T^{-1}(\tilde{X})$ and $Y=T^{-1}(\tilde{Y})$. Then $(X, Y)$ is a coupling between $v$ and $\mu \star \gamma_{1}$ and it holds

$$
\mathbb{E}[\tilde{k}(X, Y)]=\mathbb{E}[\rho(T(X), T(Y))]=\mathbb{E}[\rho(\tilde{X}, \tilde{Y})]=\mathcal{T}_{\rho}(\tilde{\nu}, \tilde{\mu}) \leq H(\tilde{v} \mid \tilde{\mu})=H\left(\nu \mid \mu \star \gamma_{1}\right),
$$

where the last equality comes from the fact that if $\nu \ll \mu \star \gamma_{1}$, then $\tilde{v} \ll \tilde{\mu}$ with

$$
\frac{d \tilde{\nu}}{d \tilde{\mu}}(u)=\frac{d v}{d\left(\mu \star \gamma_{1}\right)}\left(T^{-1}(u)\right), \quad \forall u \in \mathbb{R}^{d}
$$

This concludes the first step.

Step 2. A lower bound on the cost function $\tilde{k}$. We now bound $\tilde{k}(x, y)$ from below by the more convenient cost function $k(x, y)$. According to Lemma 2.6 of [10], $|\omega(u)-\omega(v)| \geq \omega(|u-v| / 2)$, for all $u, v \in \mathbb{R}$. Therefore, for all $x, y$ in $\mathbb{R}^{d}$ :

$$
\begin{aligned}
|T(x)-T(y)|^{2} & =\sum_{i}\left|\omega\left(x_{i}\right)-\omega\left(y_{i}\right)\right|^{2} \\
& \geq \sum_{i} \omega\left(\frac{\left|x_{i}-y_{i}\right|}{2}\right)^{2} \\
& =\sum_{i}\left(\frac{1}{2}\left|x_{i}-y_{i}\right|+\frac{1}{8}\left|x_{i}-y_{i}\right|^{2}\right)^{2} \\
& \geq \frac{1}{4} \sum_{i}\left|x_{i}-y_{i}\right|^{2}+\frac{1}{64} \sum_{i}\left|x_{i}-y_{i}\right|^{4} \\
& \geq \frac{1}{32}\left(\frac{1}{2}|x-y|^{2}+\frac{1}{2}\|x-y\|_{4}^{4}\right)
\end{aligned}
$$

Using the inequality $\alpha(a u) \geq \alpha(a) \alpha(u)$ for all $a, u \in \mathbb{R}$ ([10], Lemma 2.6) and the concavity of the function $u \mapsto \alpha(\sqrt{u}), u \in \mathbb{R}^{+}$, this leads to the following bound on the cost function $\tilde{k}$ :

$$
\begin{aligned}
\tilde{k}(x, y) & \geq \alpha\left(\frac{1}{D \sqrt{32}}\left(\frac{1}{2}|x-y|^{2}+\frac{1}{2}\|x-y\|_{4}^{4}\right)^{1 / 2}\right) \\
& \geq \frac{1}{2} \alpha\left(\frac{1}{D \sqrt{32}}\right)\left(\alpha(|x-y|)+\alpha\left(\|x-y\|_{4}^{2}\right)\right) .
\end{aligned}
$$

Finally, it is easy to check that $\alpha\left(\frac{1}{D \sqrt{32}}\right) \geq \frac{c^{\prime \prime \prime}}{C(R)}$ for some universal constant $c^{\prime \prime \prime}$, which completes the proof. 
Remark 5. If one could improve the conclusion in the result by Bobkov, Gentil, Ledoux and conclude that $\tilde{\mu}$ satisfies the transport inequality with the cost function

$$
(x, y) \mapsto \sum_{i=1}^{d} \alpha\left(\frac{1}{D}\left|x_{i}-y_{i}\right|\right)
$$

instead of $\rho$, then one would conclude that $\mu$ satisfies Talagrand's inequality, with respect to the Euclidean norm, with a dimension free constant.

\section{Special cases and extensions}

\subsection{Spherically symmetric measures}

We prove in this section the following claim of Theorem 1.5:

Theorem 4.1. If $\mu$ is a spherically symmetric measure with support in $B_{d}(0, R)$, then $\mu \star \gamma_{\delta}$ satisfies a logarithmic Sobolev inequality and

$$
C_{L S}\left(\mu \star \gamma_{\delta}\right) \leq 4 \delta^{2} \exp \left(\frac{8}{\pi} \frac{R^{2}}{\delta^{2}}\right) .
$$

Let us recall that $\mu \star \gamma_{\delta}$ is the law of the random variable $S=X+\delta Z$. By assumption, the law $\mu$ of $X$ is spherically symmetric, that is, invariant by any vectorial rotation of $\mathbb{R}^{d}$. Since $Z$ has the same invariance, this implies that the density $p(z)$ of $S$ only depends on the norm of $z$, thus we can write:

$$
p(z)=p\left(|z| e_{1}\right)=\int_{\mathbb{R}^{d}} \frac{1}{\left(2 \pi \delta^{2}\right)^{d / 2}} \exp \left(-\frac{1}{2 \delta^{2}}\left(\left(|z|-x_{1}\right)^{2}+\sum_{i=2}^{d} x_{i}^{2}\right)\right) d \mu\left(x_{1}, x_{2}, \ldots, x_{d}\right) .
$$

Denoting, for all $r \in \mathbb{R}$,

$$
\hat{p}_{\delta}(r)=\int_{\mathbb{R}} \frac{1}{\left(2 \pi \delta^{2}\right)^{1 / 2}} \exp \left(-\frac{\left(|z|-x_{1}\right)^{2}}{2 \delta^{2}}\right) d \hat{\mu}_{1}\left(x_{1}\right)
$$

the density of the convolution of $\gamma_{\delta}$ with the first marginal $\hat{\mu}_{1}$ of the measure

$$
\frac{1}{\left(2 \pi \delta^{2}\right)^{(d-1) / 2}} \exp \left(-\frac{1}{2 \delta^{2}} \sum_{i=2}^{d} x_{i}^{2}\right) d \mu\left(x_{1}, x_{2}, \ldots, x_{d}\right)
$$

one has $p(z)=\hat{p}_{\delta}(|z|)$.

Since the one-dimensional measure $\hat{\mu}_{1}$ is supported in the interval $[-R, R]$, the method from Section 2.2 apply. Using Lemma 2.1, with $\sigma=2 R a_{1}$, we obtain a decomposition

$$
-\log \left(\hat{p}_{\delta}(r)\right)=w_{\sigma}(r)+w_{b}(r)
$$


where $w_{\sigma}: \mathbb{R} \rightarrow \mathbb{R}$ is $1 /\left(2 \delta^{2}\right)$-convex and $w_{b}: \mathbb{R} \rightarrow \mathbb{R}$ is bounded by $2\left(R a_{1} / \delta\right)^{2}$.

Since the measure $\hat{\mu}_{1}$ is symmetric, the function $\hat{p}_{\delta}$ is even, so that $w_{\sigma}$ and $w_{b}$ constructed in the proof of Lemma 2.1 are even too.

This entails a decomposition of $p$ on $\mathbb{R}^{d}$ as a sum

$$
-\log (p(z))=W_{\sigma}(z)+W_{b}(r)
$$

by taking $W_{\sigma}(z)=w_{\sigma}(|z|)$ and $W_{b}(z)=w_{b}(|z|)$. The function $W_{b}$ is of course bounded by $2\left(R a_{1} / \delta\right)^{2}$. We prove in Lemma 4.2 that $W_{c}$ is convex. The conclusion follows by the same reasoning as in Section 2.2.

Lemma 4.2. Let $w: \mathbb{R} \rightarrow \mathbb{R}$ be a $\mathcal{C}^{2}$, even, and $\rho$-convex function. Then $W: \mathbb{R}^{d} \rightarrow \mathbb{R}$ defined by $W(z)=w(|z|)$ for all $z \in \mathbb{R}^{d}$ is also $\mathcal{C}^{2}$ and $\rho$-convex.

Proof. Let us denote $N(z)=|z|$. For any $z \neq 0$, one computes

$$
\begin{aligned}
\nabla N(z) & =\frac{1}{|z|} z, \\
\operatorname{Hess} N(z) & =\frac{1}{|z|}\left(I_{d}-\frac{1}{|z|^{2}} z z^{T}\right) .
\end{aligned}
$$

By composition with $w$, one deduces, for any $z \neq 0$,

$$
\begin{aligned}
\nabla W(z) & =\frac{w^{\prime}(|z|)}{|z|} z \\
\operatorname{Hess} W(z) & =\frac{w^{\prime \prime}(|z|)}{|z|^{2}} z z^{T}+\frac{w^{\prime}(|z|)}{|z|}\left(I_{d}-\frac{1}{|z|^{2}} z z^{T}\right) .
\end{aligned}
$$

These two quantities converge respectively to 0 and $w^{\prime \prime}(0) I_{d}$ when $z \rightarrow 0$. By a classical continuation lemma, this implies that $W$ is $\mathcal{C}^{2}$ with $\nabla W(0)=0$ and Hess $W(0)=w^{\prime \prime}(0) I_{d}$.

By assumption, $w^{\prime \prime}(|z|) \geq \rho$ for any $z \in \mathbb{R}^{d}$. Furthermore, for any $z \neq 0, \frac{w^{\prime}(|z|)}{|z|} \geq \rho$ (since the assumptions imply that 0 is a minimum of $w)$. Finally, noting that $z z^{T}$ and $\left(I_{d}-\frac{1}{|z|^{2}} z z^{T}\right)$ are the orthogonal projections on $\operatorname{Vect}(z)$ and $z^{\perp}$, one gets that Hess $W(z) \geq \rho I_{d}$ for any $z \in \mathbb{R}^{d}$.

\subsection{Dimension free $\log -$ Sobolev for $\delta \in(R / \sqrt{2}, R)$}

The first item of Theorem 1.3 states that for $\delta>R$, the probability measure $\mu \star \gamma_{\delta}$ satisfies a logarithmic Sobolev inequality with an explicit, dimension free, constant. In this section, we improve on this result by proving the first point of Theorem 1.5.

The proof of the following result relies on the connections between functional inequalities and concentration of measure inequalities. The well-known Herbst argument shows that the logarithmic Sobolev inequality implies a Gaussian concentration of measure phenomenon. More 
precisely, if $\mu$ is a probability measure on $\mathbb{R}^{d}$ satisfying the logarithmic Sobolev inequality with a constant $C_{L S}$, then for any 1-Lipschitz function $f: \mathbb{R}^{d} \rightarrow \mathbb{R}$, it holds

$$
\mu(f \geq m+t) \leq e^{-t^{2} / C_{L S}}, \quad \forall t \geq 0,
$$

where $m=\int f d \mu$ (see, e.g., Theorem 5.3 of [13]). On the other hand, a recent result by E. Milman [17] shows that conversely under some curvature assumptions a sufficiently strong Gaussian concentration of measure inequality implies back the logarithmic Sobolev inequality. It appears that in the range of parameters $R / \sqrt{2}<\delta<R$ the measure $\mu \star \gamma_{\delta}$ is sufficiently concentrated to apply Milman's result.

Theorem 4.3. Suppose that $R / \sqrt{2}<\delta<R$, then $\mu \star \gamma_{\delta}$ satisfies a logarithmic Sobolev inequality with a constant depending only on $R$ and $\delta$ and not on $d$.

Proof. Let us examine the concentration properties of $X+\delta Z$ where $X$ and $Z$ are independent random variables with respective laws $\mu$ and $\mathcal{N}_{d}\left(0, I_{d}\right)$. If $f: \mathbb{R}^{d} \rightarrow \mathbb{R}$ is a 1-Lipschitz function, then denoting by $m=\mathbb{E}[f(X+\delta Z)]$, it holds for any $t \geq 0$

$$
\begin{aligned}
\mathbb{P}[f(X+\delta Z) \geq m+t] & =\mathbb{E}_{X}[\mathbb{P}[f(X+\delta Z) \geq m+t \mid X]] \\
& \leq \mathbb{E}_{X}\left[\exp \left(-\frac{1}{2 \delta^{2}}\left[t+m-\mathbb{E}_{Z}[f(X+\delta Z)]\right]_{+}^{2}\right)\right],
\end{aligned}
$$

where the second inequality follows from the concentration inequality satisfied by $\delta Z$ (which is for instance a consequence of the fact that $\gamma_{\delta}$ satisfies the logarithmic Sobolev inequality with the constant $\left.2 \delta^{2}\right)$. Now, for any $x \in B_{d}(0, R)$,

$$
\left|m-\mathbb{E}_{Z}[f(x+\delta Z)]\right|=\left|\mathbb{E}_{X}\left[\mathbb{E}_{Z}[f(X+\delta Z)-f(x+\delta Z)]\right]\right| \leq \mathbb{E}_{X}[|X-x|] \leq 2 R .
$$

Therefore $\mathbb{E}_{Z}[f(X+\delta Z)] \leq 2 R+m$ almost surely, hence

$$
\mathbb{P}[f(X+\delta Z) \geq m+t] \leq \exp \left(-\frac{1}{2 \delta^{2}}[t-2 R]_{+}^{2}\right) .
$$

In particular, for any $0<\varepsilon<1$, it holds

$$
\mathbb{P}[f(X+\delta Z) \geq m+t] \leq \exp \left(-\frac{\varepsilon}{2 \delta^{2}} t^{2}\right), \quad \forall t>\frac{2 R}{1-\sqrt{\varepsilon}}:=t_{\varepsilon} .
$$

On the other hand, the density of the law of $X+\delta Z$ is of the form $e^{-V_{\delta}}$, with a function $V_{\delta}$ such that Hess $V_{\delta} \geq \frac{1}{\delta^{2}}-\frac{R^{2}}{\delta^{4}}=-\kappa_{\delta}$. In this range of parameters, $\kappa_{\delta}>0$. According to Theorem 1.2 of [17], as soon as $\frac{\varepsilon}{2 \delta^{2}} \geq \frac{1}{2} \kappa_{\delta}$ (which means that $R / \delta<\sqrt{1+\varepsilon}$ ), the probability measure $\mu$ satisfies a Gaussian isoperimetric inequality, which in turn implies the logarithmic Sobolev inequality with a constant depending only on the parameters $\varepsilon, R, \delta$. 


\subsection{Dimension free log-Sobolev for log-convex functions}

Recall the following results by Maurey.

Theorem 4.4 ([15], Theorem 3). Let $X$ be a bounded random variable such that $|X| \leq R$ a.s. Then $X$ satisfies the so called convex $\tau$-property:

$$
\mathbb{E}\left[e^{Q_{4 R^{2}} f(X)}\right] \mathbb{E}\left[e^{-f(X)}\right] \leq 1,
$$

for any convex function $f: \mathbb{R}^{d} \rightarrow \mathbb{R}$, where $Q_{s} f(x)=\inf _{y \in \mathbb{R}^{d}}\left\{f(y)+\frac{|x-y|^{2}}{4 s}\right\}, s>0$.

On the other hand, the Gaussian random variable $\delta Z$ with law $\mathcal{N}_{d}\left(0, \delta I_{d}\right)$ satisfies the following $\tau$-property

$$
\mathbb{E}\left[e^{Q_{\delta^{2}} f(\delta Z)}\right] \mathbb{E}\left[e^{-f(\delta Z)}\right] \leq 1,
$$

for any function $f: \mathbb{R}^{d} \rightarrow \mathbb{R}([15]$, Theorem 2$)$.

By the tensorization property of the convex $\tau$-property [15], one concludes that $(X, \delta Z)$ satisfies the following $\tau$-property

$$
\mathbb{E}\left[e^{\tilde{Q} f(X, \delta Z)}\right] \mathbb{E}\left[e^{-f(X, \delta Z)}\right] \leq 1,
$$

for any convex function $f: \mathbb{R}^{d} \times \mathbb{R}^{d} \rightarrow \mathbb{R}$, where

$$
\tilde{Q} f\left(x_{1}, x_{2}\right)=\inf _{\left(y_{1}, y_{2}\right) \in \mathbb{R}^{d} \times \mathbb{R}^{d}}\left\{f\left(y_{1}, y_{2}\right)+\frac{1}{16 R^{2}}\left|x_{1}-y_{1}\right|^{2}+\frac{1}{4 \delta^{2}}\left|x_{2}-y_{2}\right|^{2}\right\} .
$$

In particular, applying the inequality above to $f\left(x_{1}, x_{2}\right)=g\left(x_{1}+x_{2}\right)$, and using the fact that

$$
\inf _{y_{1}+y_{2}=y}\left\{\frac{1}{16 R^{2}}\left|x_{1}-y_{1}\right|^{2}+\frac{1}{4 \delta^{2}}\left|x_{2}-y_{2}\right|^{2}\right\}=\frac{1}{4 C(\delta, R)}\left|x_{1}+x_{2}-y\right|^{2},
$$

with $C(\delta, R)=\delta^{2}+4 R^{2}$, one concludes that $X+\delta Z$ satisfies

$$
\mathbb{E}\left[e^{Q_{C} g(X+\delta Z)}\right] \mathbb{E}\left[e^{-g(X+\delta Z)}\right] \leq 1,
$$

for any convex function $g: \mathbb{R}^{d} \rightarrow \mathbb{R}$.

According to [11], this inequality is equivalent to the following transport type inequality

$$
\overline{\mathcal{T}}_{2}\left(v_{1}, v_{2}\right) \leq C(\delta, R)\left(H\left(v_{1} \mid \mu \star \gamma_{\delta}\right)+H\left(v_{2} \mid \mu \star \gamma_{\delta}\right)\right)
$$

for all probability measures $v_{1}, v_{2}$ on $\mathbb{R}^{d}$, where $H\left(\cdot \mid \mu \star \gamma_{\delta}\right)$ denotes the relative entropy functional and

$$
\overline{\mathcal{T}}_{2}\left(v_{1}, v_{2}\right)=\inf _{X_{1} \sim v_{1}, X_{2} \sim v_{2}} \mathbb{E}\left[\left|X_{1}-\mathbb{E}\left[X_{2} \mid X_{1}\right]\right|^{2}\right]
$$


It is also shown in [11] that this transport inequality implies the following logarithmic Sobolev inequality

$$
\operatorname{Ent}_{\mu \star \gamma_{\delta}}\left(e^{f}\right) \leq 8\left(\delta^{2}+4 R^{2}\right) \int|\nabla f|^{2} e^{f} d \mu \star \gamma_{\delta},
$$

for any convex function $f: \mathbb{R}^{d} \rightarrow \mathbb{R}$. This proves the fourth item of Theorem 1.5.

\section{References}

[1] Aida, S. (1998). Uniform positivity improving property, Sobolev inequalities, and spectral gaps. J. Funct. Anal. 158 152-185. MR1641566

[2] Aida, S. and Shigekawa, I. (1994). Logarithmic Sobolev inequalities and spectral gaps: Perturbation theory. J. Funct. Anal. 126 448-475. MR1305076

[3] Ané, C., Blachère, S., Chafaï, D., Fougères, P., Gentil, I., Malrieu, F., Roberto, C. and Scheffer, G. (2000). Sur les Inégalités de Sobolev Logarithmiques. Panoramas et Synthèses [Panoramas and Syntheses] 10. Paris: Société Mathématique de France. With a preface by Dominique Bakry and Michel Ledoux.

[4] Bakry, D., Gentil, I. and Ledoux, M. (2014). Analysis and Geometry of Markov Diffusion Operators. Grundlehren der Mathematischen Wissenschaften [Fundamental Principles of Mathematical Sciences] 348. Cham: Springer. MR3155209

[5] Bobkov, S.G., Gentil, I. and Ledoux, M. (2001). Hypercontractivity of Hamilton-Jacobi equations. J. Math. Pures Appl. (9) 80 669-696. MR1846020

[6] Bobkov, S.G. and Götze, F. (1999). Exponential integrability and transportation cost related to logarithmic Sobolev inequalities. J. Funct. Anal. 163 1-28. MR1682772

[7] Cattiaux, P., Guillin, A. and Wu, L.-M. (2010). A note on Talagrand's transportation inequality and logarithmic Sobolev inequality. Probab. Theory Related Fields 148 285-304. MR2653230

[8] Chafaï, D. and Malrieu, F. (2010). On fine properties of mixtures with respect to concentration of measure and Sobolev type inequalities. Ann. Inst. Henri Poincaré Probab. Stat. 46 72-96. MR2641771

[9] Diaconis, P. and Saloff-Coste, L. (1996). Logarithmic Sobolev inequalities for finite Markov chains. Ann. Appl. Probab. 6 695-750. MR1410112

[10] Gozlan, N. (2010). Poincaré inequalities and dimension free concentration of measure. Ann. Inst. Henri Poincaré Probab. Stat. 46 708-739. MR2682264

[11] Gozlan, N., Roberto, C., Samson, P.-M. and Tetali, P. (2014). Kantorovich duality for general transport costs and applications. Preprint. Available at arXiv:1412.7480.

[12] Houdré, C. (2001). Mixed and isoperimetric estimates on the log-Sobolev constants of graphs and Markov chains. Combinatorica 21 489-513. MR1863575

[13] Ledoux, M. (2001). The Concentration of Measure Phenomenon. Mathematical Surveys and Monographs 89. Providence, RI: Amer. Math. Soc. MR1849347

[14] Ledoux, M. (2001). Logarithmic Sobolev inequalities for unbounded spin systems revisited. In Séminaire de Probabilités XXXV. Lecture Notes in Math. 1755 167-194. Berlin: Springer. MR1837286

[15] Maurey, B. (1991). Some deviation inequalities. Geom. Funct. Anal. 1 188-197. MR1097258

[16] Menz, G. and Schlichting, A. (2014). Poincaré and logarithmic Sobolev inequalities by decomposition of the energy landscape. Ann. Probab. 42 1809-1884. MR3262493

[17] Milman, E. (2010). Isoperimetric and concentration inequalities: Equivalence under curvature lower bound. Duke Math. J. 154 207-239. MR2682183

[18] Otto, F. and Villani, C. (2000). Generalization of an inequality by Talagrand and links with the logarithmic Sobolev inequality. J. Funct. Anal. 173 361-400. MR1760620 
[19] Royer, G. (2007). An Initiation to Logarithmic Sobolev Inequalities. SMF/AMS Texts and Monographs 14. Providence, RI: Amer. Math. Soc.; Paris: Société Mathématique de France. Translated from the 1999 French original by Donald Babbitt. MR2352327

[20] Schlichting, A. (2012). The Eyring-Kramers formula for Poincaré and logarithmic Sobolev inequalities Ph.D. thesis, Leipzig University.

[21] Wang, F.-Y. and Wang, J. (2016). Functional inequalities for convolution probability measures. Ann. Inst. Henri Poincaré Probab. Stat. 52 898-914. MR3498015

[22] Zimmermann, D. (2013). Logarithmic Sobolev inequalities for mollified compactly supported measures. J. Funct. Anal. 265 1064-1083. MR3067796

[23] Zimmermann, D. (2014). Bounds for logarithmic Sobolev constants for Gaussian convolutions of compactly supported measures. Preprint. Available at arxiv:1405.2581.

[24] Zimmermann, D. (2016). Elementary proof of logarithmic Sobolev inequalities for Gaussian convolutions on $\mathbb{R}$. Ann. Math. Blaise Pascal 23 129-140. MR3505572

Received September 2015 and revised June 2016 